\title{
Second-Order Partial Differentiation of Real Ternary Functions
}

\author{
Takao Inoué \\ Inaba 2205, Wing-Minamikan \\ Nagano, Nagano, Japan
}

\begin{abstract}
Summary. In this article, we shall extend the result of [17] to discuss second-order partial differentiation of real ternary functions (refer to [7] and [14] for partial differentiation).
\end{abstract}

MML identifier: $\underline{\text { PDIFF_5 }}$, version: $\underline{7.11 .07 \quad 4.156 .1112}$

The notation and terminology used here have been introduced in the following papers: [6], [11], [12], [1], [2], [3], [4], [5], [7], [16], [17], [13], [8], [15], [10], and $[9]$.

\section{Second-order Partial Derivatives}

For simplicity, we use the following convention: $x, x_{0}, y, y_{0}, z, z_{0}, r$ denote real numbers, $u, u_{0}$ denote elements of $\mathcal{R}^{3}, f, f_{1}, f_{2}$ denote partial functions from $\mathcal{R}^{3}$ to $\mathbb{R}, R$ denotes a rest, and $L$ denotes a linear function.

Let $f$ be a partial function from $\mathcal{R}^{3}$ to $\mathbb{R}$ and let $u$ be an element of $\mathcal{R}^{3}$. We say that $f$ is partial differentiable on 1 st-1st coordinate in $u$ if and only if the condition (Def. 1) is satisfied.

(Def. 1) There exist real numbers $x_{0}, y_{0}, z_{0}$ such that

(i) $u=\left\langle x_{0}, y_{0}, z_{0}\right\rangle$, and

(ii) there exists a neighbourhood $N$ of $x_{0}$ such that $N \subseteq$ $\operatorname{domSVF} 1(1, \operatorname{pdiff} 1(f, 1), u)$ and there exist $L, \quad R$ such that for every $x$ such that $x \in N$ holds $(\operatorname{SVF} 1(1, \operatorname{pdiff1}(f, 1), u))(x)-$ $(\operatorname{SVF} 1(1, \operatorname{pdiff1}(f, 1), u))\left(x_{0}\right)=L\left(x-x_{0}\right)+R\left(x-x_{0}\right)$. 
We say that $f$ is partial differentiable on 1st-2nd coordinate in $u$ if and only if the condition (Def. 2) is satisfied.

(Def. 2) There exist real numbers $x_{0}, y_{0}, z_{0}$ such that

(i) $u=\left\langle x_{0}, y_{0}, z_{0}\right\rangle$, and

(ii) there exists a neighbourhood $N$ of $y_{0}$ such that $N \subseteq$ $\operatorname{dom} \operatorname{SVF} 1(2, \operatorname{pdiff1}(f, 1), u)$ and there exist $L, R$ such that for every $y$ such that $y \in N$ holds $(\operatorname{SVF} 1(2, \operatorname{pdiff1}(f, 1), u))(y)-$ $(\operatorname{SVF} 1(2, \operatorname{pdiff} 1(f, 1), u))\left(y_{0}\right)=L\left(y-y_{0}\right)+R\left(y-y_{0}\right)$.

We say that $f$ is partial differentiable on 1st-3rd coordinate in $u$ if and only if the condition (Def. 3) is satisfied.

(Def. 3) There exist real numbers $x_{0}, y_{0}, z_{0}$ such that

(i) $u=\left\langle x_{0}, y_{0}, z_{0}\right\rangle$, and

(ii) there exists a neighbourhood $N$ of $z_{0}$ such that $N \subseteq$ $\operatorname{dom} \operatorname{SVF} 1(3, \operatorname{pdiff} 1(f, 1), u)$ and there exist $L, \quad R$ such that for every $z$ such that $z \in N$ holds $(\operatorname{SVF} 1(3, \operatorname{pdiff1}(f, 1), u))(z)-$ $(\operatorname{SVF} 1(3, \operatorname{pdiff1} 1(f, 1), u))\left(z_{0}\right)=L\left(z-z_{0}\right)+R\left(z-z_{0}\right)$.

We say that $f$ is partial differentiable on 2nd-1st coordinate in $u$ if and only if the condition (Def. 4) is satisfied.

(Def. 4) There exist real numbers $x_{0}, y_{0}, z_{0}$ such that

(i) $u=\left\langle x_{0}, y_{0}, z_{0}\right\rangle$, and

(ii) there exists a neighbourhood $N$ of $x_{0}$ such that $N \subseteq$ dom SVF1(1, pdiff1 $(f, 2), u)$ and there exist $L, R$ such that for every $x$ such that $x \in N$ holds $(\operatorname{SVF} 1(1, \operatorname{pdiff1}(f, 2), u))(x)-$ $(\operatorname{SVF} 1(1, \operatorname{pdiff} 1(f, 2), u))\left(x_{0}\right)=L\left(x-x_{0}\right)+R\left(x-x_{0}\right)$.

We say that $f$ is partial differentiable on 2 nd-2nd coordinate in $u$ if and only if the condition (Def. 5) is satisfied.

(Def. 5) There exist real numbers $x_{0}, y_{0}, z_{0}$ such that

(i) $u=\left\langle x_{0}, y_{0}, z_{0}\right\rangle$, and

(ii) there exists a neighbourhood $N$ of $y_{0}$ such that $N \subseteq$ $\operatorname{dom} \operatorname{SVF} 1(2, \operatorname{pdiff1}(f, 2), u)$ and there exist $L, R$ such that for every $y$ such that $y \in N$ holds $(\operatorname{SVF} 1(2, \operatorname{pdiff1}(f, 2), u))(y)-$ $(\operatorname{SVF} 1(2, \operatorname{pdiff1} 1(f, 2), u))\left(y_{0}\right)=L\left(y-y_{0}\right)+R\left(y-y_{0}\right)$.

We say that $f$ is partial differentiable on 2 nd-3rd coordinate in $u$ if and only if the condition (Def. 6) is satisfied.

(Def. 6) There exist real numbers $x_{0}, y_{0}, z_{0}$ such that

(i) $u=\left\langle x_{0}, y_{0}, z_{0}\right\rangle$, and

(ii) there exists a neighbourhood $N$ of $z_{0}$ such that $N \subseteq$ dom SVF1(3, pdiff1 $(f, 2), u)$ and there exist $L, R$ such that for every $z$ such that $z \in N$ holds $(\operatorname{SVF} 1(3, \operatorname{pdiff1}(f, 2), u))(z)-$ $(\operatorname{SVF} 1(3, \operatorname{pdiff1}(f, 2), u))\left(z_{0}\right)=L\left(z-z_{0}\right)+R\left(z-z_{0}\right)$. 
We say that $f$ is partial differentiable on 3rd-1st coordinate in $u$ if and only if the condition (Def. 7) is satisfied.

(Def. 7) There exist real numbers $x_{0}, y_{0}, z_{0}$ such that

(i) $u=\left\langle x_{0}, y_{0}, z_{0}\right\rangle$, and

(ii) there exists a neighbourhood $N$ of $x_{0}$ such that $N \subseteq$ $\operatorname{domSVF} 1(1, \operatorname{pdiff} 1(f, 3), u)$ and there exist $L, \quad R$ such that for every $x$ such that $x \in N$ holds $(\operatorname{SVF} 1(1, \operatorname{pdiff} 1(f, 3), u))(x)-$ $(\operatorname{SVF} 1(1, \operatorname{pdiff} 1(f, 3), u))\left(x_{0}\right)=L\left(x-x_{0}\right)+R\left(x-x_{0}\right)$.

We say that $f$ is partial differentiable on 3 rd-2nd coordinate in $u$ if and only if the condition (Def. 8) is satisfied.

(Def. 8) There exist real numbers $x_{0}, y_{0}, z_{0}$ such that

(i) $u=\left\langle x_{0}, y_{0}, z_{0}\right\rangle$, and

(ii) there exists a neighbourhood $N$ of $y_{0}$ such that $N \subseteq$ $\operatorname{domSVF} 1(2, \operatorname{pdiff} 1(f, 3), u)$ and there exist $L, \quad R$ such that for every $y$ such that $y \in N$ holds $(\operatorname{SVF} 1(2, \operatorname{pdiff1}(f, 3), u))(y)-$ $(\operatorname{SVF} 1(2, \operatorname{pdiff} 1(f, 3), u))\left(y_{0}\right)=L\left(y-y_{0}\right)+R\left(y-y_{0}\right)$.

We say that $f$ is partial differentiable on 3rd-3rd coordinate in $u$ if and only if the condition (Def. 9) is satisfied.

(Def. 9) There exist real numbers $x_{0}, y_{0}, z_{0}$ such that

(i) $u=\left\langle x_{0}, y_{0}, z_{0}\right\rangle$, and

(ii) there exists a neighbourhood $N$ of $z_{0}$ such that $N \subseteq$ $\operatorname{domSVF} 1(3, \operatorname{pdiff1}(f, 3), u)$ and there exist $L, R$ such that for every $z$ such that $z \in N$ holds $(\operatorname{SVF} 1(3, \operatorname{pdiff1}(f, 3), u))(z)-$ $(\operatorname{SVF} 1(3, \operatorname{pdiff} 1(f, 3), u))\left(z_{0}\right)=L\left(z-z_{0}\right)+R\left(z-z_{0}\right)$.

Let $f$ be a partial function from $\mathcal{R}^{3}$ to $\mathbb{R}$ and let $u$ be an element of $\mathcal{R}^{3}$. Let us assume that $f$ is partial differentiable on 1st-1st coordinate in $u$. The functor hpartdiff11 $(f, u)$ yielding a real number is defined by the condition (Def. 10).

(Def. 10) There exist real numbers $x_{0}, y_{0}, z_{0}$ such that

(i) $u=\left\langle x_{0}, y_{0}, z_{0}\right\rangle$, and

(ii) there exists a neighbourhood $N$ of $x_{0}$ such that $N \subseteq$ $\operatorname{dom} \operatorname{SVF} 1(1, \operatorname{pdiff} 1(f, 1), u)$ and there exist $L, R$ such that hpartdiff11 $(f, u)=$ $L(1)$ and for every $x$ such that $x \in N$ holds $(\operatorname{SVF} 1(1, \operatorname{pdiff} 1(f, 1), u))(x)-$ $(\operatorname{SVF} 1(1, \operatorname{pdiff} 1(f, 1), u))\left(x_{0}\right)=L\left(x-x_{0}\right)+R\left(x-x_{0}\right)$.

Let $f$ be a partial function from $\mathcal{R}^{3}$ to $\mathbb{R}$ and let $u$ be an element of $\mathcal{R}^{3}$. Let us assume that $f$ is partial differentiable on 1st-2nd coordinate in $u$. The functor hpartdiff12 $(f, u)$ yielding a real number is defined by the condition (Def. 11).

(Def. 11) There exist real numbers $x_{0}, y_{0}, z_{0}$ such that

(i) $u=\left\langle x_{0}, y_{0}, z_{0}\right\rangle$, and

(ii) there exists a neighbourhood $N$ of $y_{0}$ such that $N \subseteq$ $\operatorname{dom} \operatorname{SVF} 1(2, \operatorname{pdiff} 1(f, 1), u)$ and there exist $L, R$ such that hpartdiff12 $(f, u)=$ 
$L(1)$ and for every $y$ such that $y \in N$ holds $(\operatorname{SVF} 1(2, \operatorname{pdiff} 1(f, 1), u))(y)-$ $(\operatorname{SVF} 1(2, \operatorname{pdiff} 1(f, 1), u))\left(y_{0}\right)=L\left(y-y_{0}\right)+R\left(y-y_{0}\right)$.

Let $f$ be a partial function from $\mathcal{R}^{3}$ to $\mathbb{R}$ and let $u$ be an element of $\mathcal{R}^{3}$. Let us assume that $f$ is partial differentiable on 1st-3rd coordinate in $u$. The functor hpartdiff13 $(f, u)$ yielding a real number is defined by the condition (Def. 12).

(Def. 12) There exist real numbers $x_{0}, y_{0}, z_{0}$ such that

(i) $u=\left\langle x_{0}, y_{0}, z_{0}\right\rangle$, and

(ii) there exists a neighbourhood $N$ of $z_{0}$ such that $N \subseteq$ $\operatorname{dom} \operatorname{SVF} 1(3, \operatorname{pdiff} 1(f, 1), u)$ and there exist $L, R$ such that hpartdiff13 $(f, u)=$ $L(1)$ and for every $z$ such that $z \in N$ holds $(\operatorname{SVF} 1(3, \operatorname{pdiff} 1(f, 1), u))(z)-$ $(\operatorname{SVF} 1(3, \operatorname{pdiff} 1(f, 1), u))\left(z_{0}\right)=L\left(z-z_{0}\right)+R\left(z-z_{0}\right)$.

Let $f$ be a partial function from $\mathcal{R}^{3}$ to $\mathbb{R}$ and let $u$ be an element of $\mathcal{R}^{3}$. Let us assume that $f$ is partial differentiable on 2nd-1st coordinate in $u$. The functor hpartdiff21 $(f, u)$ yielding a real number is defined by the condition (Def. 13).

(Def. 13) There exist real numbers $x_{0}, y_{0}, z_{0}$ such that

(i) $u=\left\langle x_{0}, y_{0}, z_{0}\right\rangle$, and

(ii) there exists a neighbourhood $N$ of $x_{0}$ such that $N \subseteq$ $\operatorname{dom} \operatorname{SVF} 1(1, \operatorname{pdiff} 1(f, 2), u)$ and there exist $L, R$ such that hpartdiff21 $(f, u)=$ $L(1)$ and for every $x$ such that $x \in N$ holds $(\operatorname{SVF} 1(1, \operatorname{pdiff} 1(f, 2), u))(x)-$ $(\operatorname{SVF} 1(1, \operatorname{pdiff} 1(f, 2), u))\left(x_{0}\right)=L\left(x-x_{0}\right)+R\left(x-x_{0}\right)$.

Let $f$ be a partial function from $\mathcal{R}^{3}$ to $\mathbb{R}$ and let $u$ be an element of $\mathcal{R}^{3}$. Let us assume that $f$ is partial differentiable on 2 nd-2nd coordinate in $u$. The functor hpartdiff22 $(f, u)$ yielding a real number is defined by the condition (Def. 14).

(Def. 14) There exist real numbers $x_{0}, y_{0}, z_{0}$ such that

(i) $u=\left\langle x_{0}, y_{0}, z_{0}\right\rangle$, and

(ii) there exists a neighbourhood $N$ of $y_{0}$ such that $N \subseteq$ $\operatorname{dom} \operatorname{SVF} 1(2, \operatorname{pdiff} 1(f, 2), u)$ and there exist $L, R$ such that hpartdiff22 $(f, u)=$ $L(1)$ and for every $y$ such that $y \in N$ holds $(\operatorname{SVF} 1(2, \operatorname{pdiff} 1(f, 2), u))(y)-$ $(\operatorname{SVF} 1(2, \operatorname{pdiff} 1(f, 2), u))\left(y_{0}\right)=L\left(y-y_{0}\right)+R\left(y-y_{0}\right)$.

Let $f$ be a partial function from $\mathcal{R}^{3}$ to $\mathbb{R}$ and let $u$ be an element of $\mathcal{R}^{3}$. Let us assume that $f$ is partial differentiable on 2nd-3rd coordinate in $u$. The functor hpartdiff23 $(f, u)$ yielding a real number is defined by the condition (Def. 15).

(Def. 15) There exist real numbers $x_{0}, y_{0}, z_{0}$ such that

(i) $u=\left\langle x_{0}, y_{0}, z_{0}\right\rangle$, and

(ii) there exists a neighbourhood $N$ of $z_{0}$ such that $N \subseteq$ $\operatorname{dom} \operatorname{SVF} 1(3, \operatorname{pdiff1}(f, 2), u)$ and there exist $L, R$ such that hpartdiff23 $(f, u)=$ $L(1)$ and for every $z$ such that $z \in N$ holds $(\operatorname{SVF} 1(3, \operatorname{pdiff1}(f, 2), u))(z)-$ $(\operatorname{SVF} 1(3, \operatorname{pdiff} 1(f, 2), u))\left(z_{0}\right)=L\left(z-z_{0}\right)+R\left(z-z_{0}\right)$.

Let $f$ be a partial function from $\mathcal{R}^{3}$ to $\mathbb{R}$ and let $u$ be an element of $\mathcal{R}^{3}$. Let us assume that $f$ is partial differentiable on $3 \mathrm{rd}-1$ st coordinate in $u$. The functor 
hpartdiff31 $(f, u)$ yields a real number and is defined by the condition (Def. 16).

(Def. 16) There exist real numbers $x_{0}, y_{0}, z_{0}$ such that

(i) $u=\left\langle x_{0}, y_{0}, z_{0}\right\rangle$, and

(ii) there exists a neighbourhood $N$ of $x_{0}$ such that $N \subseteq$ $\operatorname{dom} \operatorname{SVF} 1(1, \operatorname{pdiff1}(f, 3), u)$ and there exist $L, R$ such that hpartdiff31 $(f, u)=$ $L(1)$ and for every $x$ such that $x \in N$ holds $(\operatorname{SVF} 1(1, \operatorname{pdiff} 1(f, 3), u))(x)-$ $(\operatorname{SVF} 1(1, \operatorname{pdiff1}(f, 3), u))\left(x_{0}\right)=L\left(x-x_{0}\right)+R\left(x-x_{0}\right)$.

Let $f$ be a partial function from $\mathcal{R}^{3}$ to $\mathbb{R}$ and let $u$ be an element of $\mathcal{R}^{3}$. Let us assume that $f$ is partial differentiable on 3 rd-2nd coordinate in $u$. The functor hpartdiff32 $(f, u)$ yielding a real number is defined by the condition (Def. 17).

(Def. 17) There exist real numbers $x_{0}, y_{0}, z_{0}$ such that

(i) $u=\left\langle x_{0}, y_{0}, z_{0}\right\rangle$, and

(ii) there exists a neighbourhood $N$ of $y_{0}$ such that $N \subseteq$ $\operatorname{dom} \operatorname{SVF} 1(2, \operatorname{pdiff1}(f, 3), u)$ and there exist $L, R$ such that hpartdiff32 $(f, u)=$ $L(1)$ and for every $y$ such that $y \in N$ holds $(\operatorname{SVF} 1(2, \operatorname{pdiff} 1(f, 3), u))(y)-$ $(\operatorname{SVF} 1(2, \operatorname{pdiff} 1(f, 3), u))\left(y_{0}\right)=L\left(y-y_{0}\right)+R\left(y-y_{0}\right)$.

Let $f$ be a partial function from $\mathcal{R}^{3}$ to $\mathbb{R}$ and let $u$ be an element of $\mathcal{R}^{3}$. Let us assume that $f$ is partial differentiable on $3 \mathrm{rd}-3 \mathrm{rd}$ coordinate in $u$. The functor hpartdiff33 $(f, u)$ yielding a real number is defined by the condition (Def. 18).

(Def. 18) There exist real numbers $x_{0}, y_{0}, z_{0}$ such that

(i) $u=\left\langle x_{0}, y_{0}, z_{0}\right\rangle$, and

(ii) there exists a neighbourhood $N$ of $z_{0}$ such that $N \subseteq$ $\operatorname{dom} \operatorname{SVF} 1(3, \operatorname{pdiff1}(f, 3), u)$ and there exist $L, R$ such that hpartdiff33 $(f, u)=$ $L(1)$ and for every $z$ such that $z \in N$ holds $(\operatorname{SVF} 1(3, \operatorname{pdiff} 1(f, 3), u))(z)-$ $(\operatorname{SVF} 1(3, \operatorname{pdiff1}(f, 3), u))\left(z_{0}\right)=L\left(z-z_{0}\right)+R\left(z-z_{0}\right)$.

Next we state a number of propositions:

(1) If $u=\left\langle x_{0}, y_{0}, z_{0}\right\rangle$ and $f$ is partial differentiable on 1st-1st coordinate in $u$, then $\operatorname{SVF} 1(1, \operatorname{pdiff} 1(f, 1), u)$ is differentiable in $x_{0}$.

(2) If $u=\left\langle x_{0}, y_{0}, z_{0}\right\rangle$ and $f$ is partial differentiable on 1st-2nd coordinate in $u$, then $\operatorname{SVF} 1(2, \operatorname{pdiff} 1(f, 1), u)$ is differentiable in $y_{0}$.

(3) If $u=\left\langle x_{0}, y_{0}, z_{0}\right\rangle$ and $f$ is partial differentiable on 1st-3rd coordinate in $u$, then $\operatorname{SVF} 1(3, \operatorname{pdiff} 1(f, 1), u)$ is differentiable in $z_{0}$.

(4) If $u=\left\langle x_{0}, y_{0}, z_{0}\right\rangle$ and $f$ is partial differentiable on 2nd-1st coordinate in $u$, then $\operatorname{SVF} 1(1, \operatorname{pdiff} 1(f, 2), u)$ is differentiable in $x_{0}$.

(5) If $u=\left\langle x_{0}, y_{0}, z_{0}\right\rangle$ and $f$ is partial differentiable on 2nd-2nd coordinate in $u$, then $\operatorname{SVF} 1(2, \operatorname{pdiff} 1(f, 2), u)$ is differentiable in $y_{0}$.

(6) If $u=\left\langle x_{0}, y_{0}, z_{0}\right\rangle$ and $f$ is partial differentiable on 2nd-3rd coordinate in $u$, then $\operatorname{SVF} 1(3, \operatorname{pdiff} 1(f, 2), u)$ is differentiable in $z_{0}$.

(7) If $u=\left\langle x_{0}, y_{0}, z_{0}\right\rangle$ and $f$ is partial differentiable on 3rd-1st coordinate in $u$, then $\operatorname{SVF} 1(1, \operatorname{pdiff} 1(f, 3), u)$ is differentiable in $x_{0}$. 
(8) If $u=\left\langle x_{0}, y_{0}, z_{0}\right\rangle$ and $f$ is partial differentiable on 3rd-2nd coordinate in $u$, then $\operatorname{SVF} 1(2, \operatorname{pdiff} 1(f, 3), u)$ is differentiable in $y_{0}$.

(9) If $u=\left\langle x_{0}, y_{0}, z_{0}\right\rangle$ and $f$ is partial differentiable on 3rd-3rd coordinate in $u$, then $\operatorname{SVF} 1(3, \operatorname{pdiff} 1(f, 3), u)$ is differentiable in $z_{0}$.

(10) If $u=\left\langle x_{0}, y_{0}, z_{0}\right\rangle$ and $f$ is partial differentiable on 1st-1st coordinate in $u$, then $\operatorname{hpartdiff1} 1(f, u)=(\operatorname{SVF} 1(1, \operatorname{pdiff} 1(f, 1), u))^{\prime}\left(x_{0}\right)$.

(11) If $u=\left\langle x_{0}, y_{0}, z_{0}\right\rangle$ and $f$ is partial differentiable on 1st-2nd coordinate in $u$, then hpartdiff12 $(f, u)=(\operatorname{SVF} 1(2, \operatorname{pdiff} 1(f, 1), u))^{\prime}\left(y_{0}\right)$.

(12) If $u=\left\langle x_{0}, y_{0}, z_{0}\right\rangle$ and $f$ is partial differentiable on 1st-3rd coordinate in $u$, then $\operatorname{hpartdiff} 13(f, u)=(\operatorname{SVF} 1(3, \operatorname{pdiff} 1(f, 1), u))^{\prime}\left(z_{0}\right)$.

(13) If $u=\left\langle x_{0}, y_{0}, z_{0}\right\rangle$ and $f$ is partial differentiable on 2nd-1st coordinate in $u$, then $\operatorname{hpartdiff} 21(f, u)=(\operatorname{SVF} 1(1, \operatorname{pdiff} 1(f, 2), u))^{\prime}\left(x_{0}\right)$.

(14) If $u=\left\langle x_{0}, y_{0}, z_{0}\right\rangle$ and $f$ is partial differentiable on 2nd-2nd coordinate in $u$, then hpartdiff22 $(f, u)=(\operatorname{SVF} 1(2, \operatorname{pdiff} 1(f, 2), u))^{\prime}\left(y_{0}\right)$.

(15) If $u=\left\langle x_{0}, y_{0}, z_{0}\right\rangle$ and $f$ is partial differentiable on 2nd-3rd coordinate in $u$, then hpartdiff23 $(f, u)=(\operatorname{SVF} 1(3, \operatorname{pdiff} 1(f, 2), u))^{\prime}\left(z_{0}\right)$.

(16) If $u=\left\langle x_{0}, y_{0}, z_{0}\right\rangle$ and $f$ is partial differentiable on 3rd-1st coordinate in $u$, then $\operatorname{hpartdiff3} 1(f, u)=(\operatorname{SVF} 1(1, \operatorname{pdiff} 1(f, 3), u))^{\prime}\left(x_{0}\right)$.

(17) If $u=\left\langle x_{0}, y_{0}, z_{0}\right\rangle$ and $f$ is partial differentiable on 3rd-2nd coordinate in $u$, then hpartdiff32 $(f, u)=(\operatorname{SVF} 1(2, \operatorname{pdiff} 1(f, 3), u))^{\prime}\left(y_{0}\right)$.

(18) If $u=\left\langle x_{0}, y_{0}, z_{0}\right\rangle$ and $f$ is partial differentiable on 3rd-3rd coordinate in $u$, then $\operatorname{hpartdiff33}(f, u)=(\operatorname{SVF} 1(3, \operatorname{pdiff} 1(f, 3), u))^{\prime}\left(z_{0}\right)$.

Let $f$ be a partial function from $\mathcal{R}^{3}$ to $\mathbb{R}$ and let $D$ be a set. We say that $f$ is partial differentiable on 1st-1st coordinate on $D$ if and only if:

(Def. 19) $D \subseteq \operatorname{dom} f$ and for every element $u$ of $\mathcal{R}^{3}$ such that $u \in D$ holds $f\lceil D$ is partial differentiable on 1st-1st coordinate in $u$.

We say that $f$ is partial differentiable on 1 st-2nd coordinate on $D$ if and only if:

(Def. 20) $D \subseteq \operatorname{dom} f$ and for every element $u$ of $\mathcal{R}^{3}$ such that $u \in D$ holds $f\lceil D$ is partial differentiable on 1st-2nd coordinate in $u$.

We say that $f$ is partial differentiable on 1st-3rd coordinate on $D$ if and only if: (Def. 21) $D \subseteq \operatorname{dom} f$ and for every element $u$ of $\mathcal{R}^{3}$ such that $u \in D$ holds $f\lceil D$ is partial differentiable on 1st-3rd coordinate in $u$.

We say that $f$ is partial differentiable on 2 nd-1st coordinate on $D$ if and only if:

(Def. 22) $\quad D \subseteq \operatorname{dom} f$ and for every element $u$ of $\mathcal{R}^{3}$ such that $u \in D$ holds $f\lceil D$ is partial differentiable on 2nd-1st coordinate in $u$.

We say that $f$ is partial differentiable on 2 nd-2nd coordinate on $D$ if and only if:

(Def. 23) $D \subseteq \operatorname{dom} f$ and for every element $u$ of $\mathcal{R}^{3}$ such that $u \in D$ holds $f\lceil D$ is partial differentiable on 2nd-2nd coordinate in $u$.

We say that $f$ is partial differentiable on 2 nd-3rd coordinate on $D$ if and only if: 
(Def. 24) $D \subseteq \operatorname{dom} f$ and for every element $u$ of $\mathcal{R}^{3}$ such that $u \in D$ holds $f\lceil D$ is partial differentiable on 2nd-3rd coordinate in $u$.

We say that $f$ is partial differentiable on 3rd-1st coordinate on $D$ if and only if:

(Def. 25) $D \subseteq \operatorname{dom} f$ and for every element $u$ of $\mathcal{R}^{3}$ such that $u \in D$ holds $f\lceil D$ is partial differentiable on 3rd-1st coordinate in $u$.

We say that $f$ is partial differentiable on 3rd-2nd coordinate on $D$ if and only if:

(Def. 26) $D \subseteq \operatorname{dom} f$ and for every element $u$ of $\mathcal{R}^{3}$ such that $u \in D$ holds $f\lceil D$ is partial differentiable on 3rd-2nd coordinate in $u$.

We say that $f$ is partial differentiable on 3rd-3rd coordinate on $D$ if and only if:

(Def. 27) $D \subseteq \operatorname{dom} f$ and for every element $u$ of $\mathcal{R}^{3}$ such that $u \in D$ holds $f\lceil D$ is partial differentiable on 3rd-3rd coordinate in $u$.

Let $f$ be a partial function from $\mathcal{R}^{3}$ to $\mathbb{R}$ and let $D$ be a set. Let us assume that $f$ is partial differentiable on 1 st-1st coordinate on $D$. The functor $f_{\lceil D}^{1 \text { st-1st }}$ yields a partial function from $\mathcal{R}^{3}$ to $\mathbb{R}$ and is defined by:

(Def. 28) $\operatorname{dom}\left(f_{\ulcorner D}^{1 \text { st-1st }}\right)=D$ and for every element $u$ of $\mathcal{R}^{3}$ such that $u \in D$ holds $f_{\lceil D}^{1 \text { st }-1 \text { st }}(u)=\operatorname{hpartdiff} 11(f, u)$.

Let $f$ be a partial function from $\mathcal{R}^{3}$ to $\mathbb{R}$ and let $D$ be a set. Let us assume that $f$ is partial differentiable on 1 st-2nd coordinate on $D$. The functor $f_{\uparrow D}^{1 \text { st-2nd }}$ yielding a partial function from $\mathcal{R}^{3}$ to $\mathbb{R}$ is defined by:

(Def. 29) $\operatorname{dom}\left(f_{\uparrow D}^{1 \text { st-2nd }}\right)=D$ and for every element $u$ of $\mathcal{R}^{3}$ such that $u \in D$ holds $f_{\lceil D}^{1 \text { st }-2 \text { nd }}(u)=$ hpartdiff $12(f, u)$.

Let $f$ be a partial function from $\mathcal{R}^{3}$ to $\mathbb{R}$ and let $D$ be a set. Let us assume that $f$ is partial differentiable on 1 st-3rd coordinate on $D$. The functor $f_{\uparrow D}^{1 \text { st-3rd }}$ yields a partial function from $\mathcal{R}^{3}$ to $\mathbb{R}$ and is defined by:

(Def. 30) $\operatorname{dom}\left(f_{\uparrow D}^{1 \text { st-3rd }}\right)=D$ and for every element $u$ of $\mathcal{R}^{3}$ such that $u \in D$ holds $f_{\lceil D}^{1 \text { st }-3 \text { rd }}(u)=\operatorname{hpartdiff} 13(f, u)$.

Let $f$ be a partial function from $\mathcal{R}^{3}$ to $\mathbb{R}$ and let $D$ be a set. Let us assume that $f$ is partial differentiable on 2 nd-1st coordinate on $D$. The functor $f_{\uparrow D}^{2 \text { nd }-1 \text { st }}$ yielding a partial function from $\mathcal{R}^{3}$ to $\mathbb{R}$ is defined as follows:

(Def. 31) $\operatorname{dom}\left(f_{\uparrow D}^{2 \text { nd }-1 \mathrm{st}}\right)=D$ and for every element $u$ of $\mathcal{R}^{3}$ such that $u \in D$ holds $f_{\lceil D}^{2 \text { nd }-1 \text { st }}(u)=$ hpartdiff $21(f, u)$.

Let $f$ be a partial function from $\mathcal{R}^{3}$ to $\mathbb{R}$ and let $D$ be a set. Let us assume that $f$ is partial differentiable on 2 nd-2nd coordinate on $D$. The functor $f_{\uparrow D}^{2 \text { nd }-2 \text { nd }}$ yields a partial function from $\mathcal{R}^{3}$ to $\mathbb{R}$ and is defined by:

(Def. 32) $\operatorname{dom}\left(f_{\uparrow D}^{2 \text { nd }-2 \text { nd }}\right)=D$ and for every element $u$ of $\mathcal{R}^{3}$ such that $u \in D$ holds $f_{\uparrow D}^{2 \text { nd }-2 \text { nd }}(u)=\operatorname{hpartdiff} 22(f, u)$.

Let $f$ be a partial function from $\mathcal{R}^{3}$ to $\mathbb{R}$ and let $D$ be a set. Let us assume that $f$ is partial differentiable on 2 nd-3rd coordinate on $D$. The functor $f_{\uparrow D}^{2 \text { nd-3rd }}$ 
yields a partial function from $\mathcal{R}^{3}$ to $\mathbb{R}$ and is defined by:

(Def. 33) $\operatorname{dom}\left(f_{\lceil D}^{2 \text { nd }-3 \text { rd }}\right)=D$ and for every element $u$ of $\mathcal{R}^{3}$ such that $u \in D$ holds $f_{\lceil D}^{2 \mathrm{nd}-3 \mathrm{rd}}(u)=\operatorname{hpartdiff} 23(f, u)$.

Let $f$ be a partial function from $\mathcal{R}^{3}$ to $\mathbb{R}$ and let $D$ be a set. Let us assume that $f$ is partial differentiable on 3 rd-1st coordinate on $D$. The functor $f_{\Gamma D}^{3 \mathrm{rd}-1 \text { st }}$ yields a partial function from $\mathcal{R}^{3}$ to $\mathbb{R}$ and is defined as follows:

(Def. 34) $\operatorname{dom}\left(f_{\lceil D}^{3 \mathrm{rd}-1 \mathrm{st}}\right)=D$ and for every element $u$ of $\mathcal{R}^{3}$ such that $u \in D$ holds $f_{\lceil D}^{3 \mathrm{rd}-1 \mathrm{st}}(u)=\operatorname{hpartdiff} 31(f, u)$.

Let $f$ be a partial function from $\mathcal{R}^{3}$ to $\mathbb{R}$ and let $D$ be a set. Let us assume that $f$ is partial differentiable on 3 rd-2nd coordinate on $D$. The functor $f_{\lceil D}^{3 \text { rd-2nd }}$ yields a partial function from $\mathcal{R}^{3}$ to $\mathbb{R}$ and is defined by:

(Def. 35) $\operatorname{dom}\left(f_{\lceil D}^{3 \mathrm{rd}-2 \mathrm{nd}}\right)=D$ and for every element $u$ of $\mathcal{R}^{3}$ such that $u \in D$ holds $f_{\lceil D}^{3 \mathrm{rd}-2 \mathrm{nd}}(u)=\operatorname{hpartdiff3} 2(f, u)$.

Let $f$ be a partial function from $\mathcal{R}^{3}$ to $\mathbb{R}$ and let $D$ be a set. Let us assume that $f$ is partial differentiable on 3 rd-3rd coordinate on $D$. The functor $f_{\lceil D}^{3 \mathrm{rd}-3 \mathrm{rd}}$ yielding a partial function from $\mathcal{R}^{3}$ to $\mathbb{R}$ is defined by:

(Def. 36) $\operatorname{dom}\left(f_{\lceil D}^{3 \mathrm{rd}-3 \mathrm{rd}}\right)=D$ and for every element $u$ of $\mathcal{R}^{3}$ such that $u \in D$ holds $f_{\lceil D}^{3 \mathrm{rd}-3 \mathrm{rd}}(u)=\operatorname{hpartdiff3} 3(f, u)$.

\section{Main Properties of Second-order Partial Derivatives}

Next we state a number of propositions:

(19) $f$ is partial differentiable on 1st-1st coordinate in $u$ if and only if $\operatorname{pdiff} 1(f, 1)$ is partially differentiable in $u$ w.r.t. 1 .

(20) $f$ is partial differentiable on 1st-2nd coordinate in $u$ if and only if $\operatorname{pdiff} 1(f, 1)$ is partially differentiable in $u$ w.r.t. 2 .

(21) $f$ is partial differentiable on 1st-3rd coordinate in $u$ if and only if $\operatorname{pdiff} 1(f, 1)$ is partially differentiable in $u$ w.r.t. 3.

(22) $f$ is partial differentiable on 2nd-1st coordinate in $u$ if and only if $\operatorname{pdiff} 1(f, 2)$ is partially differentiable in $u$ w.r.t. 1 .

(23) $f$ is partial differentiable on 2 nd-2nd coordinate in $u$ if and only if $\operatorname{pdiff} 1(f, 2)$ is partially differentiable in $u$ w.r.t. 2 .

(24) $f$ is partial differentiable on 2nd-3rd coordinate in $u$ if and only if $\operatorname{pdiff} 1(f, 2)$ is partially differentiable in $u$ w.r.t. 3 .

(25) $f$ is partial differentiable on 3rd-1st coordinate in $u$ if and only if $\operatorname{pdiff} 1(f, 3)$ is partially differentiable in $u$ w.r.t. 1 .

(26) $f$ is partial differentiable on 3rd-2nd coordinate in $u$ if and only if $\operatorname{pdiff} 1(f, 3)$ is partially differentiable in $u$ w.r.t. 2 . 
(27) $f$ is partial differentiable on 3rd-3rd coordinate in $u$ if and only if $\operatorname{pdiff} 1(f, 3)$ is partially differentiable in $u$ w.r.t. 3 .

(28) If $f$ is partial differentiable on 1st-1st coordinate in $u$, then $\operatorname{hpartdiff} 11(f, u)=\operatorname{partdiff}(\operatorname{pdiff} 1(f, 1), u, 1)$.

(29) If $f$ is partial differentiable on 1st-2nd coordinate in $u$, then hpartdiff12 $(f, u)=\operatorname{partdiff}(\operatorname{pdiff1}(f, 1), u, 2)$.

(30) If $f$ is partial differentiable on 1st-3rd coordinate in $u$, then $\operatorname{hpartdiff13}(f, u)=\operatorname{partdiff}(\operatorname{pdiff} 1(f, 1), u, 3)$.

(31) If $f$ is partial differentiable on 2nd-1st coordinate in $u$, then hpartdiff21 $(f, u)=\operatorname{partdiff}(\operatorname{pdiff} 1(f, 2), u, 1)$.

(32) If $f$ is partial differentiable on 2nd-2nd coordinate in $u$, then hpartdiff $22(f, u)=\operatorname{partdiff}(\operatorname{pdiff} 1(f, 2), u, 2)$.

(33) If $f$ is partial differentiable on 2nd-3rd coordinate in $u$, then $\operatorname{hpartdiff23}(f, u)=\operatorname{partdiff}(\operatorname{pdiff} 1(f, 2), u, 3)$.

(34) If $f$ is partial differentiable on 3rd-1st coordinate in $u$, then $\operatorname{hpartdiff} 31(f, u)=\operatorname{partdiff}(\operatorname{pdifff} 1(f, 3), u, 1)$.

(35) If $f$ is partial differentiable on 3rd-2nd coordinate in $u$, then hpartdiff32 $(f, u)=\operatorname{partdiff}(\operatorname{pdiff} 1(f, 3), u, 2)$.

(36) If $f$ is partial differentiable on 3rd-3rd coordinate in $u$, then $\operatorname{hpartdiff33}(f, u)=\operatorname{partdiff}(\operatorname{pdiff} 1(f, 3), u, 3)$.

(37) Let $u_{0}$ be an element of $\mathcal{R}^{3}$ and $N$ be a neighbourhood of $(\operatorname{proj}(1,3))\left(u_{0}\right)$. Suppose $f$ is partial differentiable on 1st-1st coordinate in $u_{0}$ and $N \subseteq \operatorname{domSVF} 1\left(1, \operatorname{pdiff} 1(f, 1), u_{0}\right)$. Let $h$ be a convergent to 0 sequence of real numbers and $c$ be a constant sequence of real numbers. Suppose $\operatorname{rng} c=\left\{(\operatorname{proj}(1,3))\left(u_{0}\right)\right\}$ and $\operatorname{rng}(h+c) \subseteq N$. Then $h^{-1}\left(\left(\operatorname{SVF} 1\left(1, \operatorname{pdiff} 1(f, 1), u_{0}\right)_{*}(h+c)\right)-\left(\operatorname{SVF} 1\left(1, \operatorname{pdifff} 1(f, 1), u_{0}\right)_{*} c\right)\right)$ is convergent and hpartdiff11 $\left(f, u_{0}\right)=\lim \left(h^{-1}\left(\left(\operatorname{SVF} 1\left(1, \operatorname{pdiff1}(f, 1), u_{0}\right)_{*}(h+\right.\right.\right.$ $\left.\left.c))-\left(\operatorname{SVF} 1\left(1, \operatorname{pdiff} 1(f, 1), u_{0}\right)_{*} c\right)\right)\right)$.

(38) Let $u_{0}$ be an element of $\mathcal{R}^{3}$ and $N$ be a neighbourhood of $(\operatorname{proj}(2,3))\left(u_{0}\right)$. Suppose $f$ is partial differentiable on 1st-2nd coordinate in $u_{0}$ and $N \subseteq \operatorname{domSVF} 1\left(2, \operatorname{pdiff} 1(f, 1), u_{0}\right)$. Let $h$ be a convergent to 0 sequence of real numbers and $c$ be a constant sequence of real numbers. Suppose $\operatorname{rng} c=\left\{(\operatorname{proj}(2,3))\left(u_{0}\right)\right\}$ and $\operatorname{rng}(h+c) \subseteq N$. Then $h^{-1}\left(\left(\operatorname{SVF} 1\left(2, \operatorname{pdiff} 1(f, 1), u_{0}\right)_{*}(h+c)\right)-\left(\operatorname{SVF} 1\left(2, \operatorname{pdiff} 1(f, 1), u_{0}\right)_{*} c\right)\right)$ is convergent and hpartdiff12 $\left(f, u_{0}\right)=\lim \left(h^{-1}\left(\left(\operatorname{SVF} 1\left(2, \operatorname{pdiff1}(f, 1), u_{0}\right)_{*}(h+\right.\right.\right.$ $\left.\left.c))-\left(\operatorname{SVF} 1\left(2, \operatorname{pdiff} 1(f, 1), u_{0}\right)_{*} c\right)\right)\right)$.

(39) Let $u_{0}$ be an element of $\mathcal{R}^{3}$ and $N$ be a neighbourhood of $(\operatorname{proj}(3,3))\left(u_{0}\right)$. Suppose $f$ is partial differentiable on 1st-3rd coordinate in $u_{0}$ and $N \subseteq \operatorname{domSVF} 1\left(3, \operatorname{pdiff1}(f, 1), u_{0}\right)$. Let $h$ be a convergent to 0 sequence of real numbers and $c$ be a constant sequence of real num- 
bers. Suppose $\operatorname{rng} c=\left\{(\operatorname{proj}(3,3))\left(u_{0}\right)\right\}$ and $\operatorname{rng}(h+c) \subseteq N$. Then $h^{-1}\left(\left(\operatorname{SVF} 1\left(3, \operatorname{pdiff} 1(f, 1), u_{0}\right)_{*}(h+c)\right)-\left(\operatorname{SVF} 1\left(3, \operatorname{pdiff1} 1(f, 1), u_{0}\right)_{*} c\right)\right)$ is convergent and hpartdiff13 $\left(f, u_{0}\right)=\lim \left(h^{-1}\left(\left(\operatorname{SVF} 1\left(3, \operatorname{pdiff} 1(f, 1), u_{0}\right)_{*}(h+\right.\right.\right.$ $\left.\left.c))-\left(\operatorname{SVF} 1\left(3, \operatorname{pdiff} 1(f, 1), u_{0}\right)_{*} c\right)\right)\right)$.

(40) Let $u_{0}$ be an element of $\mathcal{R}^{3}$ and $N$ be a neighbourhood of $(\operatorname{proj}(1,3))\left(u_{0}\right)$. Suppose $f$ is partial differentiable on 2nd-1st coordinate in $u_{0}$ and $N \subseteq \operatorname{domSVF} 1\left(1, \operatorname{pdiff} 1(f, 2), u_{0}\right)$. Let $h$ be a convergent to 0 sequence of real numbers and $c$ be a constant sequence of real numbers. Suppose $\operatorname{rng} c=\left\{(\operatorname{proj}(1,3))\left(u_{0}\right)\right\}$ and $\operatorname{rng}(h+c) \subseteq N$. Then $h^{-1}\left(\left(\operatorname{SVF} 1\left(1, \operatorname{pdiff1} 1(f, 2), u_{0}\right)_{*}(h+c)\right)-\left(\operatorname{SVF} 1\left(1, \operatorname{pdiff} 1(f, 2), u_{0}\right)_{*} c\right)\right)$ is convergent and hpartdiff21 $\left(f, u_{0}\right)=\lim \left(h^{-1}\left(\left(\operatorname{SVF} 1\left(1, \operatorname{pdiff1}(f, 2), u_{0}\right)_{*}(h+\right.\right.\right.$ $\left.\left.c))-\left(\operatorname{SVF} 1\left(1, \operatorname{pdiff} 1(f, 2), u_{0}\right)_{*} c\right)\right)\right)$.

(41) Let $u_{0}$ be an element of $\mathcal{R}^{3}$ and $N$ be a neighbourhood of $(\operatorname{proj}(2,3))\left(u_{0}\right)$. Suppose $f$ is partial differentiable on 2 nd-2nd coordinate in $u_{0}$ and $N \subseteq \operatorname{domSVF} 1\left(2, \operatorname{pdiff} 1(f, 2), u_{0}\right)$. Let $h$ be a convergent to 0 sequence of real numbers and $c$ be a constant sequence of real numbers. Suppose $\operatorname{rng} c=\left\{(\operatorname{proj}(2,3))\left(u_{0}\right)\right\}$ and $\operatorname{rng}(h+c) \subseteq N$. Then $h^{-1}\left(\left(\operatorname{SVF} 1\left(2, \operatorname{pdiff1} 1(f, 2), u_{0}\right)_{*}(h+c)\right)-\left(\operatorname{SVF} 1\left(2, \operatorname{pdiff} 1(f, 2), u_{0}\right)_{*} c\right)\right)$ is convergent and hpartdiff22 $\left(f, u_{0}\right)=\lim \left(h^{-1}\left(\left(\operatorname{SVF} 1\left(2, \operatorname{pdiff1}(f, 2), u_{0}\right)_{*}(h+\right.\right.\right.$ $\left.\left.c))-\left(\operatorname{SVF} 1\left(2, \operatorname{pdiff} 1(f, 2), u_{0}\right)_{*} c\right)\right)\right)$.

(42) Let $u_{0}$ be an element of $\mathcal{R}^{3}$ and $N$ be a neighbourhood of $(\operatorname{proj}(3,3))\left(u_{0}\right)$. Suppose $f$ is partial differentiable on 2nd-3rd coordinate in $u_{0}$ and $N \subseteq \operatorname{domSVF} 1\left(3, \operatorname{pdiff} 1(f, 2), u_{0}\right)$. Let $h$ be a convergent to 0 sequence of real numbers and $c$ be a constant sequence of real numbers. Suppose $\operatorname{rng} c=\left\{(\operatorname{proj}(3,3))\left(u_{0}\right)\right\}$ and $\operatorname{rng}(h+c) \subseteq N$. Then $h^{-1}\left(\left(\operatorname{SVF} 1\left(3, \operatorname{pdiff} 1(f, 2), u_{0}\right)_{*}(h+c)\right)-\left(\operatorname{SVF} 1\left(3, \operatorname{pdiff} 1(f, 2), u_{0}\right)_{*} c\right)\right)$ is convergent and hpartdiff23 $\left(f, u_{0}\right)=\lim \left(h^{-1}\left(\left(\operatorname{SVF} 1\left(3, \operatorname{pdiff1}(f, 2), u_{0}\right)_{*}(h+\right.\right.\right.$ $\left.\left.c))-\left(\operatorname{SVF} 1\left(3, \operatorname{pdiff} 1(f, 2), u_{0}\right)_{*} c\right)\right)\right)$.

(43) Let $u_{0}$ be an element of $\mathcal{R}^{3}$ and $N$ be a neighbourhood of $(\operatorname{proj}(1,3))\left(u_{0}\right)$. Suppose $f$ is partial differentiable on 3rd-1st coordinate in $u_{0}$ and $N \subseteq \operatorname{domSVF} 1\left(1, \operatorname{pdiff} 1(f, 3), u_{0}\right)$. Let $h$ be a convergent to 0 sequence of real numbers and $c$ be a constant sequence of real numbers. Suppose $\operatorname{rng} c=\left\{(\operatorname{proj}(1,3))\left(u_{0}\right)\right\}$ and $\operatorname{rng}(h+c) \subseteq N$. Then $h^{-1}\left(\left(\operatorname{SVF} 1\left(1, \operatorname{pdiff1} 1(f, 3), u_{0}\right)_{*}(h+c)\right)-\left(\operatorname{SVF} 1\left(1, \operatorname{pdiff} 1(f, 3), u_{0}\right)_{*} c\right)\right)$ is convergent and hpartdiff31 $\left(f, u_{0}\right)=\lim \left(h^{-1}\left(\left(\operatorname{SVF} 1\left(1, \operatorname{pdiff1}(f, 3), u_{0}\right)_{*}(h+\right.\right.\right.$ $\left.\left.c))-\left(\operatorname{SVF} 1\left(1, \operatorname{pdiff} 1(f, 3), u_{0}\right)_{*} c\right)\right)\right)$.

(44) Let $u_{0}$ be an element of $\mathcal{R}^{3}$ and $N$ be a neighbourhood of $(\operatorname{proj}(2,3))\left(u_{0}\right)$. Suppose $f$ is partial differentiable on 3rd-2nd coordinate in $u_{0}$ and $N \subseteq \operatorname{domSVF} 1\left(2, \operatorname{pdiff} 1(f, 3), u_{0}\right)$. Let $h$ be a convergent to 0 sequence of real numbers and $c$ be a constant sequence of real numbers. Suppose $\operatorname{rng} c=\left\{(\operatorname{proj}(2,3))\left(u_{0}\right)\right\}$ and $\operatorname{rng}(h+c) \subseteq N$. Then 
$h^{-1}\left(\left(\operatorname{SVF} 1\left(2, \operatorname{pdiff} 1(f, 3), u_{0}\right)_{*}(h+c)\right)-\left(\operatorname{SVF} 1\left(2, \operatorname{pdiff} 1(f, 3), u_{0}\right)_{*} c\right)\right)$ is convergent and hpartdiff32 $\left(f, u_{0}\right)=\lim \left(h^{-1}\left(\left(\operatorname{SVF} 1\left(2, \operatorname{pdiff1}(f, 3), u_{0}\right)_{*}(h+\right.\right.\right.$ c) $\left.\left.)-\left(\operatorname{SVF} 1\left(2, \operatorname{pdiff} 1(f, 3), u_{0}\right)_{*} c\right)\right)\right)$.

(45) Let $u_{0}$ be an element of $\mathcal{R}^{3}$ and $N$ be a neighbourhood of $(\operatorname{proj}(3,3))\left(u_{0}\right)$. Suppose $f$ is partial differentiable on 3rd-3rd coordinate in $u_{0}$ and $N \subseteq \operatorname{domSVF} 1\left(3, \operatorname{pdiff} 1(f, 3), u_{0}\right)$. Let $h$ be a convergent to 0 sequence of real numbers and $c$ be a constant sequence of real numbers. Suppose $\operatorname{rng} c=\left\{(\operatorname{proj}(3,3))\left(u_{0}\right)\right\}$ and $\operatorname{rng}(h+c) \subseteq N$. Then $h^{-1}\left(\left(\operatorname{SVF} 1\left(3, \operatorname{pdiff} 1(f, 3), u_{0}\right)_{*}(h+c)\right)-\left(\operatorname{SVF} 1\left(3, \operatorname{pdiff} 1(f, 3), u_{0}\right)_{*} c\right)\right)$ is convergent and hpartdiff33 $\left(f, u_{0}\right)=\lim \left(h^{-1}\left(\left(\operatorname{SVF} 1\left(3, \operatorname{pdiff1}(f, 3), u_{0}\right)_{*}(h+\right.\right.\right.$ $\left.\left.c))-\left(\operatorname{SVF} 1\left(3, \operatorname{pdiff} 1(f, 3), u_{0}\right)_{*} c\right)\right)\right)$.

(46) Suppose that

(i) $\quad f_{1}$ is partial differentiable on 1st-1st coordinate in $u_{0}$, and

(ii) $f_{2}$ is partial differentiable on 1 st- 1 st coordinate in $u_{0}$.

Then $\operatorname{pdiff} 1\left(f_{1}, 1\right)+\operatorname{pdiff} 1\left(f_{2}, 1\right)$ is partially differentiable in $u_{0}$ w.r.t. 1 and $\operatorname{partdiff}\left(\operatorname{pdiff} 1\left(f_{1}, 1\right)+\operatorname{pdiff1}\left(f_{2}, 1\right), u_{0}, 1\right)=\operatorname{hpartdiff} 11\left(f_{1}, u_{0}\right)+$ hpartdiff11 $\left(f_{2}, u_{0}\right)$.

(47) Suppose that

(i) $\quad f_{1}$ is partial differentiable on 1 st-2nd coordinate in $u_{0}$, and

(ii) $f_{2}$ is partial differentiable on 1 st-2nd coordinate in $u_{0}$.

Then $\operatorname{pdiff} 1\left(f_{1}, 1\right)+\operatorname{pdiff} 1\left(f_{2}, 1\right)$ is partially differentiable in $u_{0}$ w.r.t. 2 and partdiff $\left(\operatorname{pdiff} 1\left(f_{1}, 1\right)+\operatorname{pdiff1}\left(f_{2}, 1\right), u_{0}, 2\right)=\operatorname{hpartdiff1} 12\left(f_{1}, u_{0}\right)+$ hpartdiff12 $\left(f_{2}, u_{0}\right)$.

(48) Suppose that

(i) $f_{1}$ is partial differentiable on 1st-3rd coordinate in $u_{0}$, and

(ii) $f_{2}$ is partial differentiable on 1st-3rd coordinate in $u_{0}$.

Then $\operatorname{pdiff} 1\left(f_{1}, 1\right)+\operatorname{pdiff} 1\left(f_{2}, 1\right)$ is partially differentiable in $u_{0}$ w.r.t. 3 and partdiff $\left(\operatorname{pdiff} 1\left(f_{1}, 1\right)+\operatorname{pdiff} 1\left(f_{2}, 1\right), u_{0}, 3\right)=\operatorname{hpartdiff} 13\left(f_{1}, u_{0}\right)+$ hpartdiff13 $\left(f_{2}, u_{0}\right)$.

(49) Suppose that

(i) $\quad f_{1}$ is partial differentiable on 2 nd-1st coordinate in $u_{0}$, and

(ii) $\quad f_{2}$ is partial differentiable on 2nd-1st coordinate in $u_{0}$.

Then $\operatorname{pdiff} 1\left(f_{1}, 2\right)+\operatorname{pdiff} 1\left(f_{2}, 2\right)$ is partially differentiable in $u_{0}$ w.r.t. 1 and partdiff( $\left.\operatorname{pdiff} 1\left(f_{1}, 2\right)+\operatorname{pdiff} 1\left(f_{2}, 2\right), u_{0}, 1\right)=\operatorname{hpartdiff} 21\left(f_{1}, u_{0}\right)+$ hpartdiff21 $\left(f_{2}, u_{0}\right)$.

(50) Suppose that

(i) $\quad f_{1}$ is partial differentiable on 2 nd-2nd coordinate in $u_{0}$, and

(ii) $f_{2}$ is partial differentiable on 2 nd-2nd coordinate in $u_{0}$.

Then $\operatorname{pdiff} 1\left(f_{1}, 2\right)+\operatorname{pdiff} 1\left(f_{2}, 2\right)$ is partially differentiable in $u_{0}$ w.r.t. 2 and partdiff $\left(\operatorname{pdiff} 1\left(f_{1}, 2\right)+\operatorname{pdiff} 1\left(f_{2}, 2\right), u_{0}, 2\right)=\operatorname{hpartdiff} 22\left(f_{1}, u_{0}\right)+$ hpartdiff22 $\left(f_{2}, u_{0}\right)$. 
(51) Suppose that

(i) $\quad f_{1}$ is partial differentiable on $2 \mathrm{nd}-3 \mathrm{rd}$ coordinate in $u_{0}$, and

(ii) $f_{2}$ is partial differentiable on 2nd-3rd coordinate in $u_{0}$.

Then $\operatorname{pdiff} 1\left(f_{1}, 2\right)+\operatorname{pdiff} 1\left(f_{2}, 2\right)$ is partially differentiable in $u_{0}$ w.r.t. 3 and partdiff $\left(\operatorname{pdiff} 1\left(f_{1}, 2\right)+\operatorname{pdiff} 1\left(f_{2}, 2\right), u_{0}, 3\right)=\operatorname{hpartdiff} 23\left(f_{1}, u_{0}\right)+$ hpartdiff23 $\left(f_{2}, u_{0}\right)$.

(52) Suppose that

(i) $\quad f_{1}$ is partial differentiable on 1st-1st coordinate in $u_{0}$, and

(ii) $f_{2}$ is partial differentiable on 1st-1st coordinate in $u_{0}$.

Then $\operatorname{pdiff} 1\left(f_{1}, 1\right)-\operatorname{pdiff} 1\left(f_{2}, 1\right)$ is partially differentiable in $u_{0}$ w.r.t. 1 and $\operatorname{partdiff}\left(\operatorname{pdiff} 1\left(f_{1}, 1\right)-\operatorname{pdiff} 1\left(f_{2}, 1\right), u_{0}, 1\right)=\operatorname{hpartdiff1} 1\left(f_{1}, u_{0}\right)-$ hpartdiff11 $\left(f_{2}, u_{0}\right)$.

(53) Suppose that

(i) $\quad f_{1}$ is partial differentiable on 1st-2nd coordinate in $u_{0}$, and

(ii) $f_{2}$ is partial differentiable on 1 st-2nd coordinate in $u_{0}$.

Then $\operatorname{pdiff} 1\left(f_{1}, 1\right)-\operatorname{pdiff} 1\left(f_{2}, 1\right)$ is partially differentiable in $u_{0}$ w.r.t. 2 and $\operatorname{partdiff}\left(\operatorname{pdiff} 1\left(f_{1}, 1\right)-\operatorname{pdiff} 1\left(f_{2}, 1\right), u_{0}, 2\right)=\operatorname{hpartdiff1} 12\left(f_{1}, u_{0}\right)-$ hpartdiff $12\left(f_{2}, u_{0}\right)$.

(54) Suppose that

(i) $\quad f_{1}$ is partial differentiable on 1 st-3rd coordinate in $u_{0}$, and

(ii) $f_{2}$ is partial differentiable on 1 st-3rd coordinate in $u_{0}$.

Then $\operatorname{pdiff} 1\left(f_{1}, 1\right)-\operatorname{pdiff} 1\left(f_{2}, 1\right)$ is partially differentiable in $u_{0}$ w.r.t. 3 and partdiff( $\left.\operatorname{pdiff1}\left(f_{1}, 1\right)-\operatorname{pdiff} 1\left(f_{2}, 1\right), u_{0}, 3\right)=\operatorname{hpartdiff} 13\left(f_{1}, u_{0}\right)-$ hpartdiff13 $\left(f_{2}, u_{0}\right)$.

(55) Suppose that

(i) $\quad f_{1}$ is partial differentiable on 2 nd-1st coordinate in $u_{0}$, and

(ii) $f_{2}$ is partial differentiable on 2 nd-1st coordinate in $u_{0}$.

Then $\operatorname{pdiff} 1\left(f_{1}, 2\right)-\operatorname{pdiff} 1\left(f_{2}, 2\right)$ is partially differentiable in $u_{0}$ w.r.t. 1 and $\operatorname{partdiff}\left(\operatorname{pdifff} 1\left(f_{1}, 2\right)-\operatorname{pdiff} 1\left(f_{2}, 2\right), u_{0}, 1\right)=\operatorname{hpartdiff2} 2\left(f_{1}, u_{0}\right)-$ hpartdiff21 $\left(f_{2}, u_{0}\right)$.

(56) Suppose that

(i) $\quad f_{1}$ is partial differentiable on $2 \mathrm{nd}-2 \mathrm{nd}$ coordinate in $u_{0}$, and

(ii) $f_{2}$ is partial differentiable on 2nd-2nd coordinate in $u_{0}$.

Then $\operatorname{pdiff} 1\left(f_{1}, 2\right)-\operatorname{pdiff} 1\left(f_{2}, 2\right)$ is partially differentiable in $u_{0}$ w.r.t. 2 and $\operatorname{partdiff}\left(\operatorname{pdiff1} 1\left(f_{1}, 2\right)-\operatorname{pdiff} 1\left(f_{2}, 2\right), u_{0}, 2\right)=\operatorname{hpartdiff} 22\left(f_{1}, u_{0}\right)-$ hpartdiff22 $\left(f_{2}, u_{0}\right)$.

(57) Suppose that

(i) $\quad f_{1}$ is partial differentiable on $2 \mathrm{nd}-3 \mathrm{rd}$ coordinate in $u_{0}$, and

(ii) $\quad f_{2}$ is partial differentiable on 2 nd-3rd coordinate in $u_{0}$.

Then $\operatorname{pdiff} 1\left(f_{1}, 2\right)-\operatorname{pdiff} 1\left(f_{2}, 2\right)$ is partially differentiable in $u_{0}$ w.r.t. 3 and partdiff $\left(\operatorname{pdifff}\left(f_{1}, 2\right)-\operatorname{pdiff} 1\left(f_{2}, 2\right), u_{0}, 3\right)=\operatorname{hpartdiff2} 2\left(f_{1}, u_{0}\right)-$ 
hpartdiff23 $\left(f_{2}, u_{0}\right)$.

(58) Suppose $f$ is partial differentiable on 1st-1st coordinate in $u_{0}$. Then $r \operatorname{pdiff} 1(f, 1)$ is partially differentiable in $u_{0}$ w.r.t. 1 and $\operatorname{partdiff}\left(r \operatorname{pdiff} 1(f, 1), u_{0}, 1\right)=r \cdot \operatorname{hpartdiff11}\left(f, u_{0}\right)$.

(59) Suppose $f$ is partial differentiable on 1st-2nd coordinate in $u_{0}$. Then $r \operatorname{pdiff} 1(f, 1)$ is partially differentiable in $u_{0}$ w.r.t. 2 and $\operatorname{partdiff}\left(r \operatorname{pdiff1}(f, 1), u_{0}, 2\right)=r \cdot \operatorname{hpartdiff1} 2\left(f, u_{0}\right)$.

(60) Suppose $f$ is partial differentiable on 1st-3rd coordinate in $u_{0}$. Then $r \operatorname{pdiff} 1(f, 1)$ is partially differentiable in $u_{0}$ w.r.t. 3 and $\operatorname{partdiff}\left(r \operatorname{pdiff} 1(f, 1), u_{0}, 3\right)=r \cdot \operatorname{hpartdiff1} 3\left(f, u_{0}\right)$.

(61) Suppose $f$ is partial differentiable on 2nd-1st coordinate in $u_{0}$. Then $r \operatorname{pdiff} 1(f, 2)$ is partially differentiable in $u_{0}$ w.r.t. 1 and $\operatorname{partdiff}\left(r \operatorname{pdiff} 1(f, 2), u_{0}, 1\right)=r \cdot \operatorname{hpartdiff} 21\left(f, u_{0}\right)$.

(62) Suppose $f$ is partial differentiable on 2nd-2nd coordinate in $u_{0}$. Then $r$ pdiffl $(f, 2)$ is partially differentiable in $u_{0}$ w.r.t. 2 and $\operatorname{partdiff}\left(r \operatorname{pdiff} 1(f, 2), u_{0}, 2\right)=r \cdot \operatorname{hpartdiff} 22\left(f, u_{0}\right)$.

(63) Suppose $f$ is partial differentiable on 2nd-3rd coordinate in $u_{0}$. Then $r \operatorname{pdiff} 1(f, 2)$ is partially differentiable in $u_{0}$ w.r.t. 3 and $\operatorname{partdiff}\left(r \operatorname{pdiff} 1(f, 2), u_{0}, 3\right)=r \cdot \operatorname{hpartdiff} 23\left(f, u_{0}\right)$.

(64) Suppose $f$ is partial differentiable on 3rd-1st coordinate in $u_{0}$. Then $r \operatorname{pdiff} 1(f, 3)$ is partially differentiable in $u_{0}$ w.r.t. 1 and $\operatorname{partdiff}\left(r \operatorname{pdiff1}(f, 3), u_{0}, 1\right)=r \cdot \operatorname{hpartdiff3}\left(f, u_{0}\right)$.

(65) Suppose $f$ is partial differentiable on 3rd-2nd coordinate in $u_{0}$. Then $r \operatorname{pdiff} 1(f, 3)$ is partially differentiable in $u_{0}$ w.r.t. 2 and $\operatorname{partdiff}\left(r \operatorname{pdiff} 1(f, 3), u_{0}, 2\right)=r \cdot \operatorname{hpartdiff32}\left(f, u_{0}\right)$.

(66) Suppose $f$ is partial differentiable on 3rd-3rd coordinate in $u_{0}$. Then $r \operatorname{pdiff} 1(f, 3)$ is partially differentiable in $u_{0}$ w.r.t. 3 and $\operatorname{partdiff}\left(r \operatorname{pdiff} 1(f, 3), u_{0}, 3\right)=r \cdot \operatorname{hpartdiff3} 3\left(f, u_{0}\right)$.

(67) Suppose that

(i) $\quad f_{1}$ is partial differentiable on 1 st-1st coordinate in $u_{0}$, and

(ii) $f_{2}$ is partial differentiable on 1st-1st coordinate in $u_{0}$.

Then $\operatorname{pdiff} 1\left(f_{1}, 1\right) \operatorname{pdiff} 1\left(f_{2}, 1\right)$ is partially differentiable in $u_{0}$ w.r.t. 1 .

(68) Suppose that

(i) $f_{1}$ is partial differentiable on 1 st-2nd coordinate in $u_{0}$, and

(ii) $f_{2}$ is partial differentiable on 1 st-2nd coordinate in $u_{0}$.

Then $\operatorname{pdiff} 1\left(f_{1}, 1\right) \operatorname{pdiff} 1\left(f_{2}, 1\right)$ is partially differentiable in $u_{0}$ w.r.t. 2 .

(69) Suppose that

(i) $\quad f_{1}$ is partial differentiable on 1st-3rd coordinate in $u_{0}$, and

(ii) $f_{2}$ is partial differentiable on 1 st-3rd coordinate in $u_{0}$.

Then $\operatorname{pdiff} 1\left(f_{1}, 1\right) \operatorname{pdiff} 1\left(f_{2}, 1\right)$ is partially differentiable in $u_{0}$ w.r.t. 3 . 
(70) Suppose that

(i) $\quad f_{1}$ is partial differentiable on 2 nd-1st coordinate in $u_{0}$, and

(ii) $f_{2}$ is partial differentiable on 2 nd-1st coordinate in $u_{0}$.

Then pdiff $1\left(f_{1}, 2\right) \operatorname{pdiff} 1\left(f_{2}, 2\right)$ is partially differentiable in $u_{0}$ w.r.t. 1 .

(71) Suppose that

(i) $\quad f_{1}$ is partial differentiable on 2 nd-2nd coordinate in $u_{0}$, and

(ii) $\quad f_{2}$ is partial differentiable on 2 nd-2nd coordinate in $u_{0}$.

Then pdiff $1\left(f_{1}, 2\right) \operatorname{pdiff} 1\left(f_{2}, 2\right)$ is partially differentiable in $u_{0}$ w.r.t. 2 .

(72) Suppose that

(i) $\quad f_{1}$ is partial differentiable on $2 \mathrm{nd}-3 \mathrm{rd}$ coordinate in $u_{0}$, and

(ii) $f_{2}$ is partial differentiable on 2nd-3rd coordinate in $u_{0}$.

Then $\operatorname{pdiff} 1\left(f_{1}, 2\right) \operatorname{pdiff} 1\left(f_{2}, 2\right)$ is partially differentiable in $u_{0}$ w.r.t. 3 .

(73) Suppose that

(i) $\quad f_{1}$ is partial differentiable on $3 r d-1$ st coordinate in $u_{0}$, and

(ii) $\quad f_{2}$ is partial differentiable on 3rd-1st coordinate in $u_{0}$.

Then $\operatorname{pdiff} 1\left(f_{1}, 3\right) \operatorname{pdiff} 1\left(f_{2}, 3\right)$ is partially differentiable in $u_{0}$ w.r.t. 1 .

(74) Suppose that

(i) $\quad f_{1}$ is partial differentiable on 3 rd-2nd coordinate in $u_{0}$, and

(ii) $\quad f_{2}$ is partial differentiable on 3rd-2nd coordinate in $u_{0}$.

Then pdiff $1\left(f_{1}, 3\right) \operatorname{pdiff} 1\left(f_{2}, 3\right)$ is partially differentiable in $u_{0}$ w.r.t. 2 .

(75) Suppose that

(i) $\quad f_{1}$ is partial differentiable on $3 \mathrm{rd}-3 \mathrm{rd}$ coordinate in $u_{0}$, and

(ii) $f_{2}$ is partial differentiable on 3rd-3rd coordinate in $u_{0}$.

Then $\operatorname{pdiff} 1\left(f_{1}, 3\right) \operatorname{pdiff} 1\left(f_{2}, 3\right)$ is partially differentiable in $u_{0}$ w.r.t. 3 .

(76) Let $u_{0}$ be an element of $\mathcal{R}^{3}$. Suppose $f$ is partial differentiable on 1 st-1st coordinate in $u_{0}$. Then $\operatorname{SVF} 1\left(1, \operatorname{pdiff} 1(f, 1), u_{0}\right)$ is continuous in $(\operatorname{proj}(1,3))\left(u_{0}\right)$.

(77) Let $u_{0}$ be an element of $\mathcal{R}^{3}$. Suppose $f$ is partial differentiable on 1 st-2nd coordinate in $u_{0}$. Then $\operatorname{SVF} 1\left(2, \operatorname{pdiff} 1(f, 1), u_{0}\right)$ is continuous in $(\operatorname{proj}(2,3))\left(u_{0}\right)$.

(78) Let $u_{0}$ be an element of $\mathcal{R}^{3}$. Suppose $f$ is partial differentiable on 1 st-3rd coordinate in $u_{0}$. Then $\operatorname{SVF} 1\left(3, \operatorname{pdiff} 1(f, 1), u_{0}\right)$ is continuous in $(\operatorname{proj}(3,3))\left(u_{0}\right)$.

(79) Let $u_{0}$ be an element of $\mathcal{R}^{3}$. Suppose $f$ is partial differentiable on 2nd-1st coordinate in $u_{0}$. Then $\operatorname{SVF} 1\left(1, \operatorname{pdiff} 1(f, 2), u_{0}\right)$ is continuous in $(\operatorname{proj}(1,3))\left(u_{0}\right)$.

(80) Let $u_{0}$ be an element of $\mathcal{R}^{3}$. Suppose $f$ is partial differentiable on 2 nd-2nd coordinate in $u_{0}$. Then $\operatorname{SVF} 1\left(2, \operatorname{pdiff} 1(f, 2), u_{0}\right)$ is continuous in $(\operatorname{proj}(2,3))\left(u_{0}\right)$. 
(81) Let $u_{0}$ be an element of $\mathcal{R}^{3}$. Suppose $f$ is partial differentiable on 2nd-3rd coordinate in $u_{0}$. Then $\operatorname{SVF} 1\left(3, \operatorname{pdiff} 1(f, 2), u_{0}\right)$ is continuous in $(\operatorname{proj}(3,3))\left(u_{0}\right)$.

(82) Let $u_{0}$ be an element of $\mathcal{R}^{3}$. Suppose $f$ is partial differentiable on 3 rd-1st coordinate in $u_{0}$. Then $\operatorname{SVF} 1\left(1, \operatorname{pdiff} 1(f, 3), u_{0}\right)$ is continuous in $(\operatorname{proj}(1,3))\left(u_{0}\right)$.

(83) Let $u_{0}$ be an element of $\mathcal{R}^{3}$. Suppose $f$ is partial differentiable on 3 rd-2nd coordinate in $u_{0}$. Then $\operatorname{SVF} 1\left(2, \operatorname{pdiff} 1(f, 3), u_{0}\right)$ is continuous in $(\operatorname{proj}(2,3))\left(u_{0}\right)$.

(84) Let $u_{0}$ be an element of $\mathcal{R}^{3}$. Suppose $f$ is partial differentiable on 3 rd-3rd coordinate in $u_{0}$. Then $\operatorname{SVF} 1\left(3, \operatorname{pdiff} 1(f, 3), u_{0}\right)$ is continuous in $(\operatorname{proj}(3,3))\left(u_{0}\right)$.

\section{REFERENCES}

[1] Grzegorz Bancerek and Krzysztof Hryniewiecki. Segments of natural numbers and finite sequences. Formalized Mathematics, 1(1):107-114, 1990.

[2] Czesław Byliński. Finite sequences and tuples of elements of a non-empty sets. Formalized Mathematics, 1(3):529-536, 1990.

[3] Czesław Byliński. Functions and their basic properties. Formalized Mathematics, 1(1):55$65,1990$.

[4] Czesław Byliński. Functions from a set to a set. Formalized Mathematics, 1(1):153-164, 1990

[5] Czesław Byliński. Partial functions. Formalized Mathematics, 1(2):357-367, 1990.

[6] Agata Darmochwał. The Euclidean space. Formalized Mathematics, 2(4):599-603, 1991.

[7] Noboru Endou, Yasunari Shidama, and Keiichi Miyajima. Partial differentiation on normed linear spaces $\mathcal{R}^{n}$. Formalized Mathematics, 15(2):65-72, 2007, doi:10.2478/v10037007-0008-5.

[8] Krzysztof Hryniewiecki. Basic properties of real numbers. Formalized Mathematics, $1(\mathbf{1}): 35-40,1990$.

[9] Jarosław Kotowicz. Convergent sequences and the limit of sequences. Formalized Mathematics, 1(2):273-275, 1990.

[10] Jarosław Kotowicz. Real sequences and basic operations on them. Formalized Mathematics, 1(2):269-272, 1990.

[11] Konrad Raczkowski and Paweł Sadowski. Real function continuity. Formalized Mathematics, 1(4):787-791, 1990.

[12] Konrad Raczkowski and Paweł Sadowski. Real function differentiability. Formalized Mathematics, 1(4):797-801, 1990.

[13] Konrad Raczkowski and Paweł Sadowski. Topological properties of subsets in real numbers. Formalized Mathematics, 1(4):777-780, 1990.

[14] Walter Rudin. Principles of Mathematical Analysis. MacGraw-Hill, 1976.

[15] Edmund Woronowicz. Relations defined on sets. Formalized Mathematics, 1(1):181-186, 1990.

[16] Bing Xie, Xiquan Liang, and Hongwei Li. Partial differentiation of real binary functions. Formalized Mathematics, 16(4):333-338, 2008, doi:10.2478/v10037-008-0041-z.

[17] Bing Xie, Xiquan Liang, and Xiuzhuan Shen. Second-order partial differentiation of real binary functions. Formalized Mathematics, 17(2):79-87, 2009, doi: 10.2478/v10037-0090009-7. 